\title{
Repeated Patterns of Dense Packings of Equal Disks in a Square
}

\author{
R. L. Graham, rlg@research.att.com \\ B. D. Lubachevsky, bdl@research.att.com \\ AT\&T Bell Laboratories \\ Murray Hill, New Jersey 07974, USA
}

Submitted: January 17, 1996; Accepted: April 20, 1996

\begin{abstract}
We examine sequences of dense packings of $n$ congruent non-overlapping disks inside a square which follow specific patterns as $n$ increases along certain values, $n=n(1), n(2), \ldots n(k), \ldots$. Extending and improving previous work of Nurmela and Östergård [NO] where previous patterns for $n=n(k)$ of the form $k^{2}, k^{2}-1, k^{2}-3, k(k+1)$, and $4 k^{2}+k$ were observed, we identify new patterns for $n=k^{2}-2$ and $n=k^{2}+\lfloor k / 2\rfloor$. We also find denser packings than those in [NO] for $n=21,28,34,40,43,44,45$, and 47 . In addition, we produce what we conjecture to be optimal packings for $n=51,52,54,55,56,60$, and 61. Finally, for each identified sequence $n(1), n(2), \ldots n(k), \ldots$ which corresponds to some specific repeated pattern, we identify a threshold index $k_{0}$, for which the packing appears to be optimal for $k \leq k_{0}$, but for which the packing is not optimal (or does not exist) for $k>k_{0}$.
\end{abstract}

\section{Introduction}

In a previous paper [GL1], the authors observed the unexpected occurrence of repeating "patterns" of dense (and presumably optimal) packings of $n$ equal non-overlapping disks inside an equilateral triangle (see Fig. 1.1 for and example). It is natural to investigate this phenomenon for other boundary shapes. In particular, this was done by the authors [LG1] for the case of $n$ disks in a circle. However, in contrast to the case of the equilateral triangle where the patterns appear to persist for arbitrarily large values of $n$, for the circle the identified packing patterns cease to be optimal as the number of disks exceeds a certain threshold.

In this note we describe the situation for the square. In a recent paper, Nurmela and Östergård [NO] present various conjectured optimal packings of $n$ equal disks in a square for up to 50 disks. They also point out certain patterns that occur there. By using a packing procedure different from theirs, we improve on their best packings for $n=21,28,34,40,43$, 44,45 , and 47 . We conjecture that these new packings are optimal, as are the new packings we give for $n=51,52,54,55,56,60$, and 61 .

We confirm all the repeated patterns mentioned in [NO], specifically, for $n=k^{2}, k^{2}-1$, $k^{2}-3$, and $k(k+1)$, and we identify two new patterns, namely, for $n=k^{2}-2$ and $n=k^{2}+\lfloor k / 2\rfloor$ The latter pattern incorporates the packings of $n=4 k^{2}+k$ disks as identified in [NO].

It was found in $[\mathrm{NO}]$ that the obvious "square" pattern of packings of $n=k^{2}$ disks becomes 


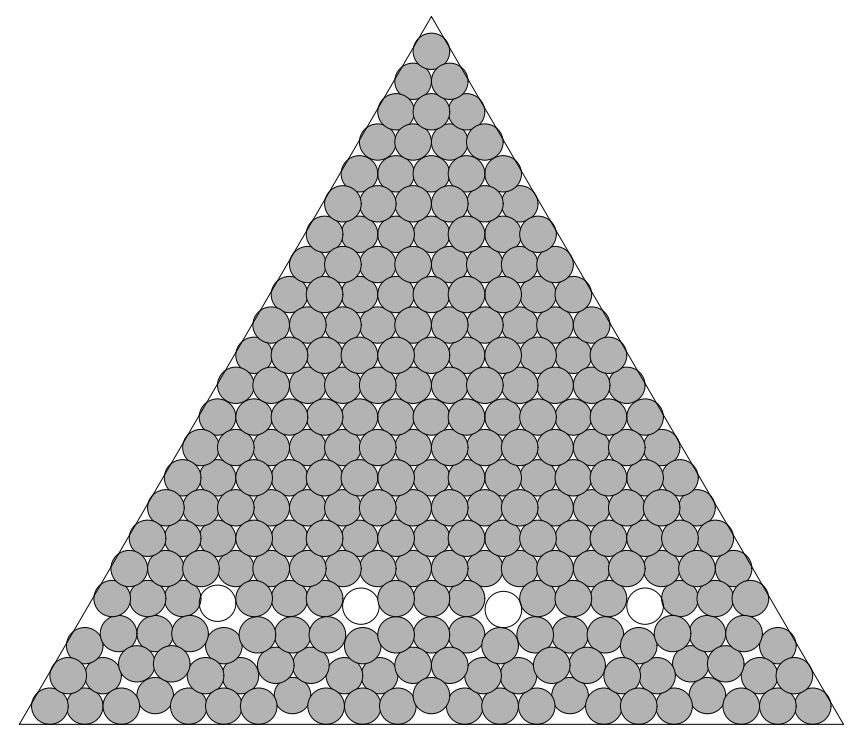

Figure 1.1: The conjectured densest packing of $n=256$ disks inside an equilateral triangle, a member of the series $n=n_{p}(k)=\Delta((k+1) p-1)+(2 p+1) \Delta(k)$ for $p=5$ and $k=3$, where $\Delta(m)=m(m+1) / 2$. The $n-p+1=252$ shaded disks can not move (they are "solid"), the $p-1=4$ non-shaded disks are free to move within their local confines (they are "rattlers"). The densest packings of $n$ disks for all checked values of the form $n=n_{p}(k), p=1,2, \ldots, k=1,2, \ldots$, have this pattern consisting of one triangle of side $(k+1) p-1$ and $2 p+1$ triangles of side $k$ with $p-1$ rattlers that are "falling off" the larger triangle.

non-optimal for $n>n_{0}=36$. This was done by presenting a configuration of $k^{2}=49$ disks with the diameters larger than $m=1 /(k-1)$. The latter $m$ is disk diameter in the packing that obeys the pattern. The standard unit of measure used in most papers on the subject is the side of the smallest square that contains the centers of disks. We repeat the same procedure for the other patterns identified both in $[\mathrm{NO}]$ and in the present paper. Namely, for each pattern we state the rule of its formation which allows us to compute the corresponding value of $m=m(n)$. Then we pinpoint the $n_{0}$ that belongs to the series and such that the packing of $n_{0}$ disks constructed according to the rule is (presumably) optimal, but for which $m\left(n_{1}\right)$ for the next value $n_{1}>n_{0}$ in the series when the packing is also constructed according to the rule is worse than a certain challenger disk configuration (which may or may not be a solid packing).

In this manner, we confirm observation in [NO] that the best packings of $n=k^{2}-1$ disks loose their characteristic pattern for $n \geq n_{1}=48$ disks. We also found that although it was not stated in [NO], the packing of 47 disks presented there (as well as our better packing of 47 disks) challenges the series $k^{2}-2$. Thus, the pattern of the series $n=k^{2}-2$ becomes non-optimal for $n>n_{0}=34$, and that of the series $n=k^{2}-1$ for $n>n_{0}=35$. We also found challenger disk configurations or packings for other patterns for values of $n>50$ which were not identified in [NO]. Namely: $n_{0}=56$ for the series $k(k+1)$ (with the challenger 
$n_{1}=72$ ), and $n_{0}=61$ for the series $k^{2}-3$ (with the challenger $n_{1}=78$ ). The situation for the series $n=n(k)=k^{2}+\lfloor k / 2\rfloor(n=5,10,18,27,39,52,68, \ldots)$ is more complex: the pattern exists for $5 \leq n(k) \leq 52$ as a solid packing and is probably optimal for these $n(k)$ except the case $n=n(3)=10$ which is the subject of several publications ([G], [Sch], [Schl], [Val]). For $n=n(8)=68$ the configuration constructed according to the pattern rule has a slight disk overlap, i.e., it does not exist as a disk packing, and the overlap persists for all $n=n(k)>68$.

In the geometric packing problem, progress in proving lags that of conjecturing. Thus, we should warn the reader that almost all our statements are conjectures; they are based on computer experimentations with the so-called "billiards" simulation algorithm [L], [LS]. In all series except $k^{2}-3$, the construction rule we found for generating a pattern for a given $n$ is a finite procedure and $m(n)$ can be expressed as the root of a well-defined polynomial. The existence of the packing of a given pattern for a fixed $n$, even if not the pattern's optimality (when appropriate), should be considered proven. However, for the series $k^{2}-3$ we have to resort on an infinite simulation procedure $[\mathrm{L}],[\mathrm{LS}]$ to construct a pattern for an arbitrary $n$ and to compute $m(n)$. Hence even the pattern's existence as a solid packing is a conjecture here. We point out that some of the proposed methods in the literature attempt to prove a packing optimal or, at the least, prove that a packing with particular parameters exists. Usually to fulfill this task, the packing must be actually presented, even if only as a conjecture. Thus, we try to present our conjectured packings in a verifiable and reproducible form; we provide 14 decimal digits of accuracy for its parameter $m$ and clearly identify the connectivity pattern (touching disk-disk and disk-wall pairs). Some previous papers provided disk coordinates in the presented packings. 1 The interested reader can contact the authors directly for the coordinates (since that would otherwise take up too much space in the paper).

\section{Packings}

The parameter $m$ supplied with each packing is the ratio of the disk diameter to the side length of the smallest square that contains the disk centers. Bonds or contact points mark disk-disk or disk-boundary contact. In the packing diagrams, bonds are indicated by black dots. Most of the packings presented are conjectures. This means that a proof is required not only for their optimality, if any, but even for their existence. Thus, a bond implies a conjecture that the corresponding distance is zero while the absence of a bond implies a conjecture that the distance is strictly positive. We placed or did not place a bond between two disks or a disk and a boundary based on the numerical evidence: the bond was placed when the corresponding distance was less than $10^{-12}$ of the disk diameter. Such a choice of a threshold is supported by the existence of a well-formed gap between a bond and a no-bond situation: In all cases when the bond is not present between apparently touching surfaces, the computed distance is at least $10^{-7}$ of the disk diameter, and, except for the packing of 47 disks in Fig.2.6, it is

\footnotetext{
${ }^{1}$ We were unable to reproduce the best packing of 21 disks for which $[\mathrm{MFP}]$ provides the diameter $m=$ 0.27181675 but no other data, e.g. no contact diagram. Our best packing of 21 disks has a smaller diameter (see Fig. 2.1).
} 
at least $10^{-5}$. The existence of this gap also testifies to that in all the packings the double precision resolution we employed for the computations sufficed. All solid disks, i.e., those that can not move, are shaded in the packing diagrams; the non-shaded disks are rattlers - they are free to move within their confines. Different shadings of disks in some packings and a unique numerical label for each disk on a diagram are provided to facilitate the discussion, These are not part of the packings. 


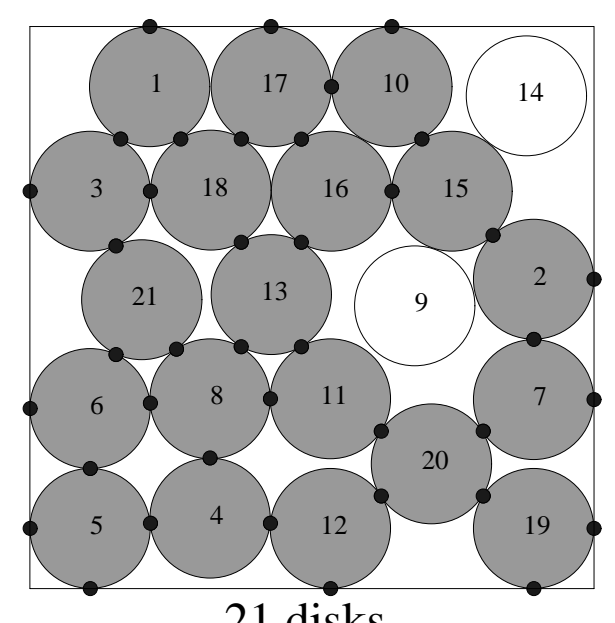

21 disks

$\mathrm{m}=0.2718122553593139$ bonds

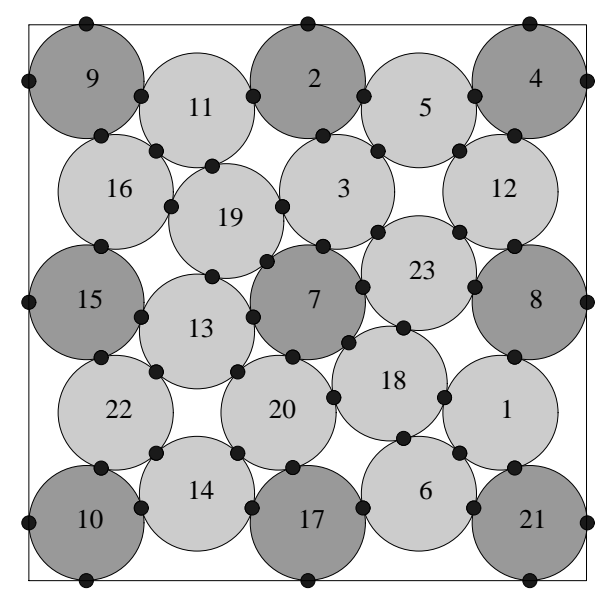

23 disks

$\mathrm{m}=0.2588190451025256$ bonds

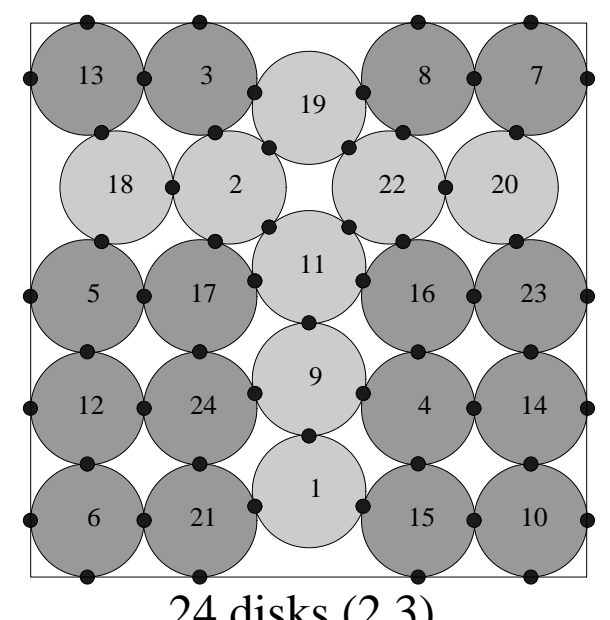

24 disks $(2,3)$

$\mathrm{m}=0.25433309503025 \quad 56$ bonds

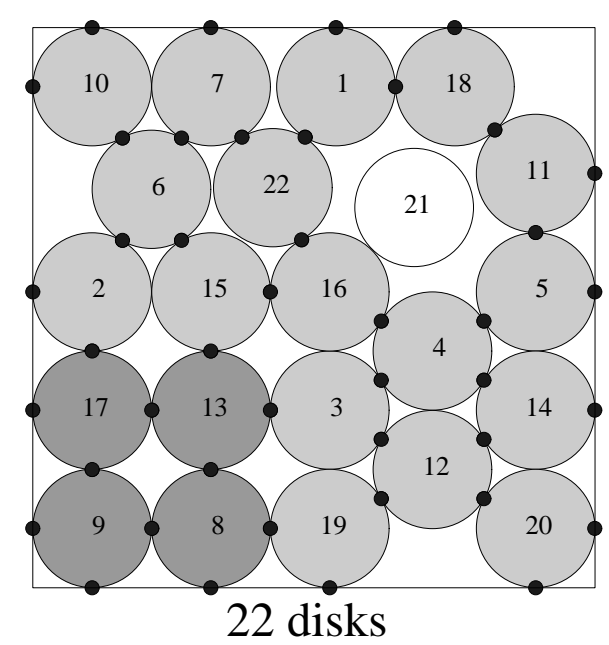

$\mathrm{m}=0.2679584015507243$ bonds

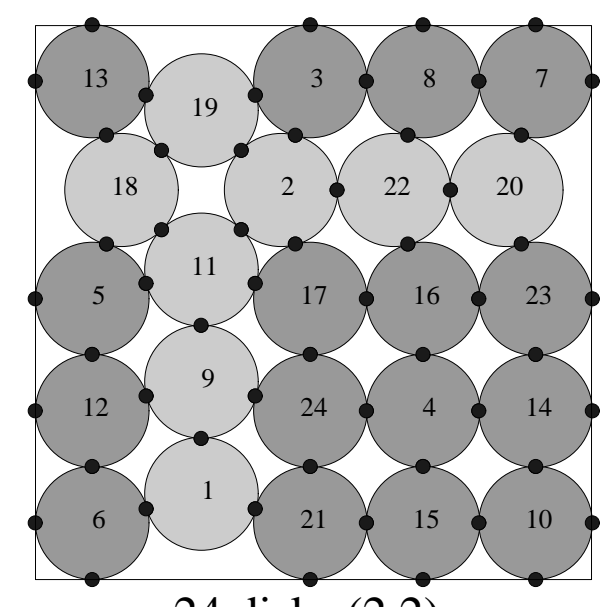

24 disks $(2,2)$

$\mathrm{m}=0.2543330950302556$ bonds

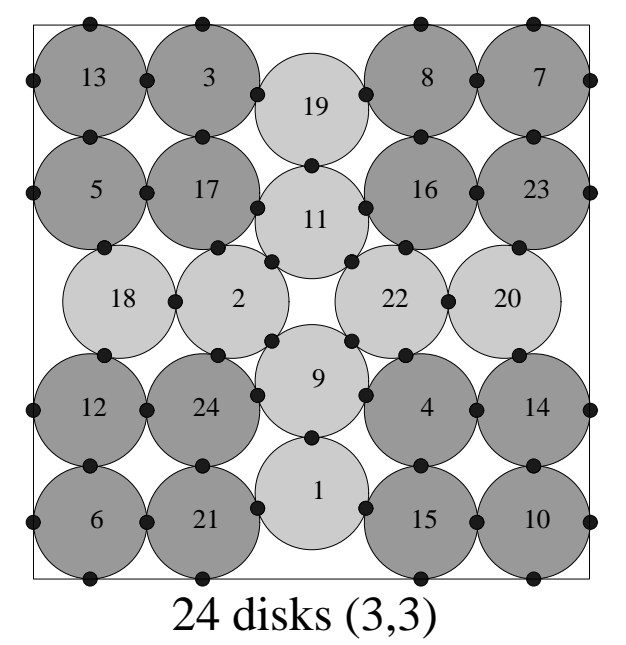

$\mathrm{m}=0.25433309503025 \quad 56$ bonds

Figure 2.1: The densest packings found of 21 to 24 disks 


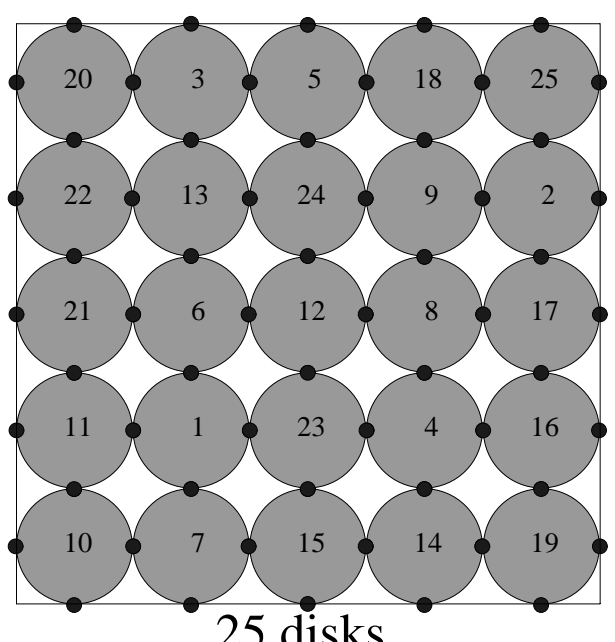

$\mathrm{m}=0.25000000000000 \quad 60$ bonds

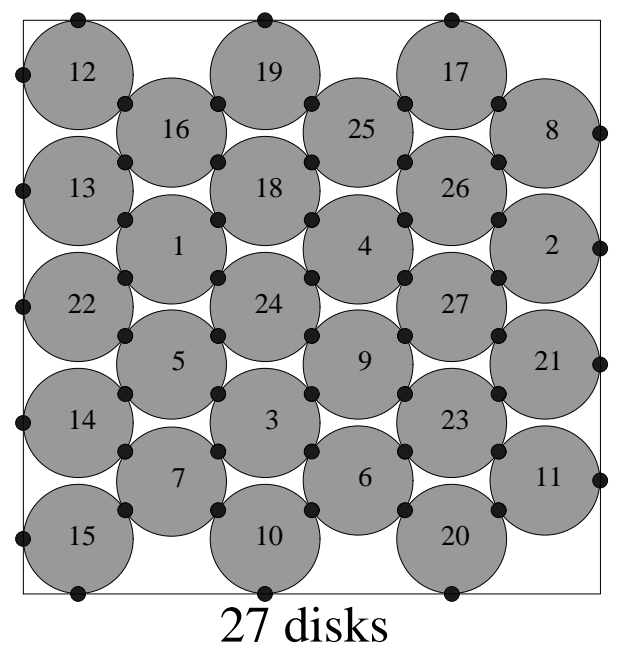

$\mathrm{m}=0.2358495283014255$ bonds

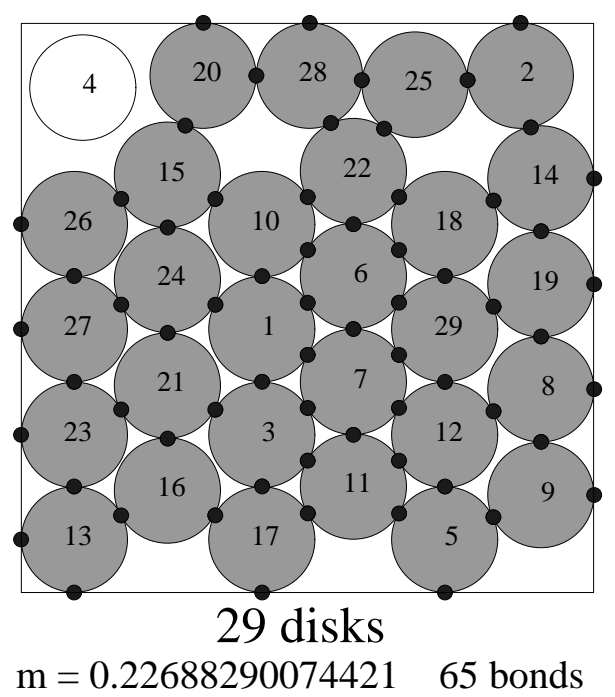

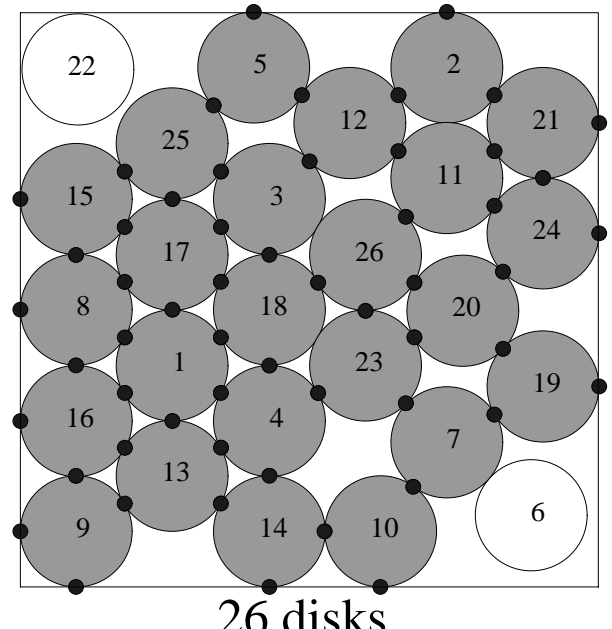
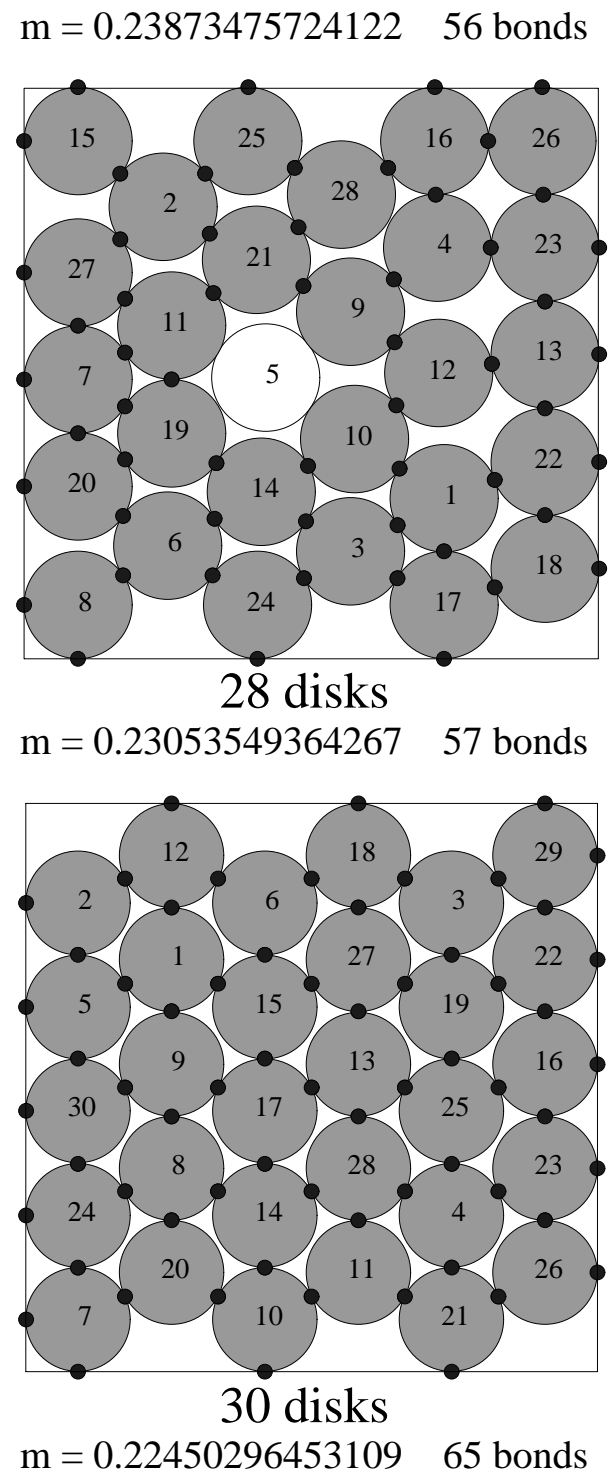

Figure 2.2: The densest packings found of 25 to 30 disks 


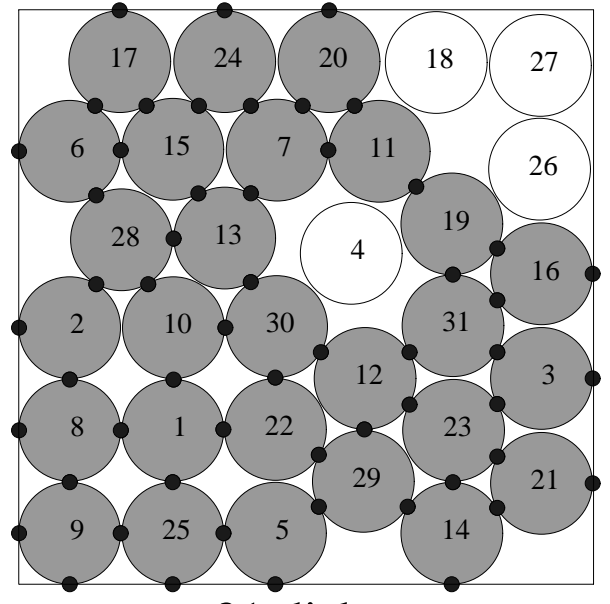

31 disks

$\mathrm{m}=0.2175472916191255$ bonds

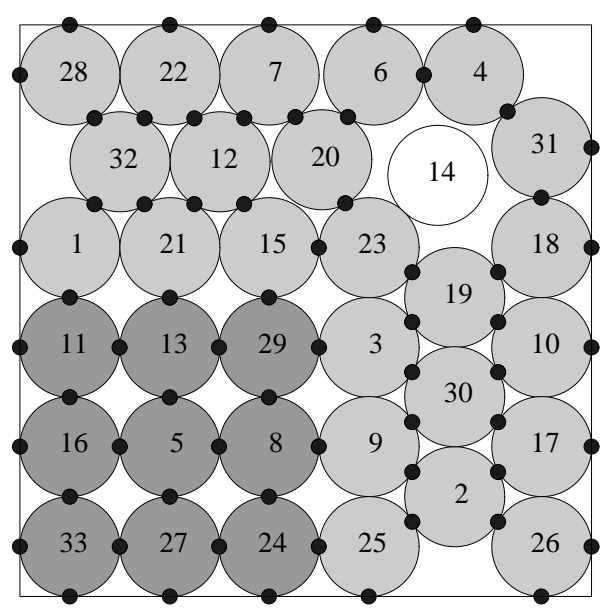

33 disks

$\mathrm{m}=0.21132838414326 \quad 65$ bonds

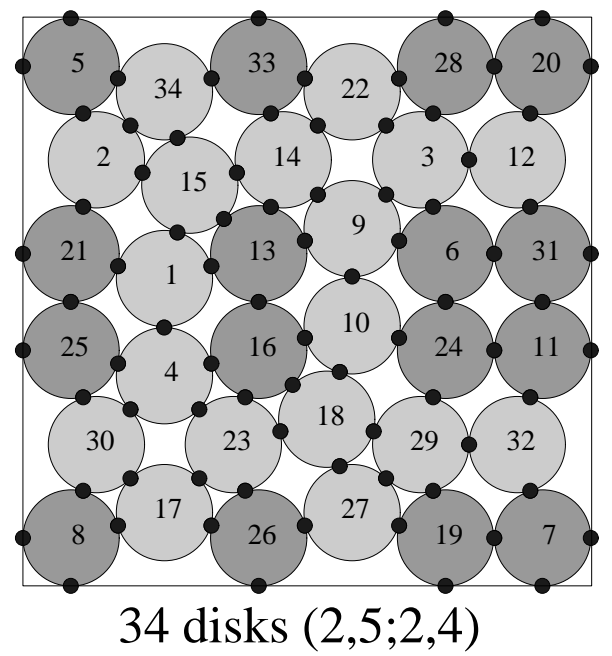

$\mathrm{m}=0.20560464675957 \quad 80$ bonds
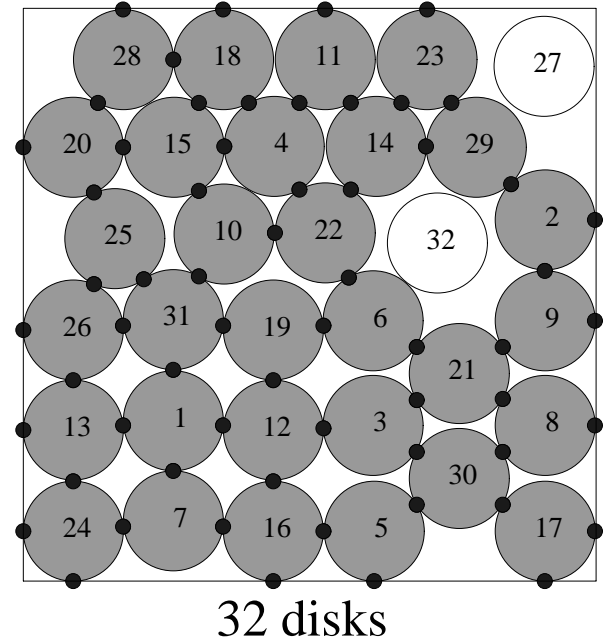

$\mathrm{m}=0.2130823529444361$ bonds

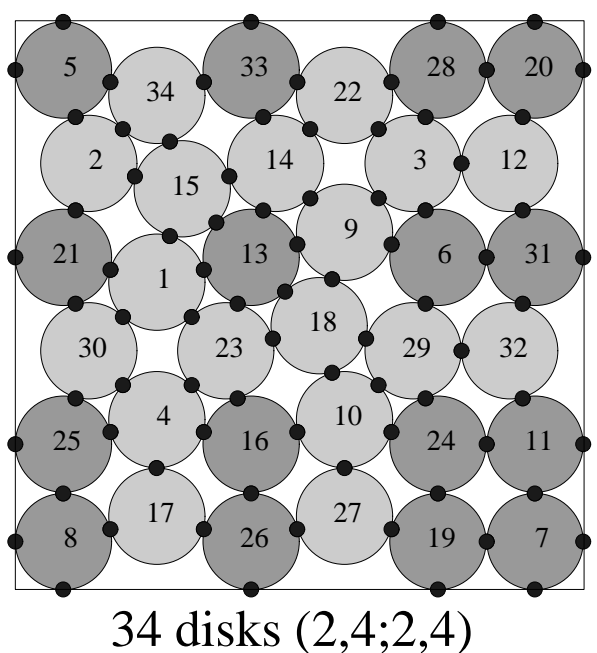

$\mathrm{m}=0.20560464675957 \quad 80$ bonds

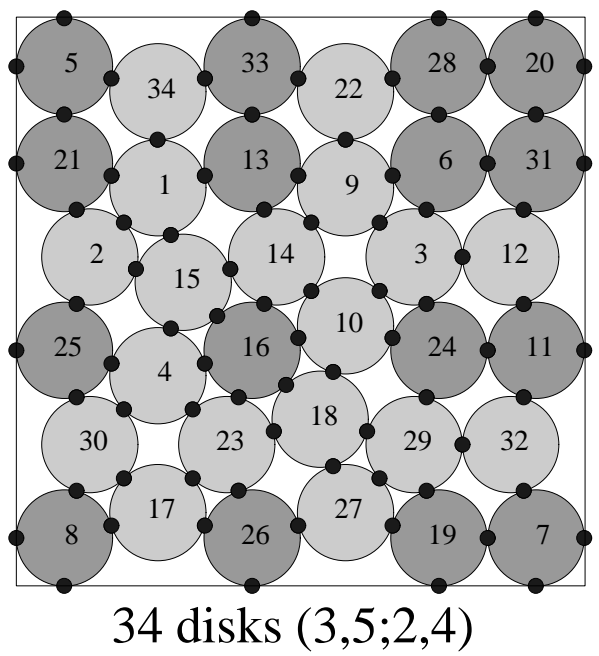

$\mathrm{m}=0.20560464675957 \quad 80$ bonds

Figure 2.3: The densest packings found of 31 to 34 disks 


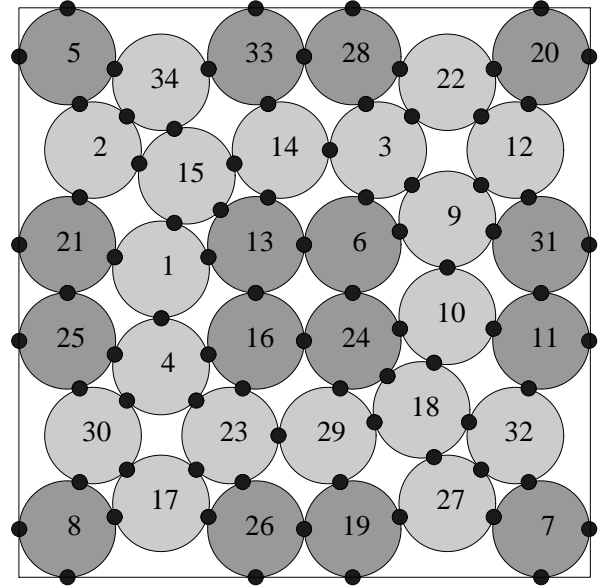

34 disks $(2,5 ; 2,5)$

$\mathrm{m}=0.20560464675957 \quad 80$ bonds

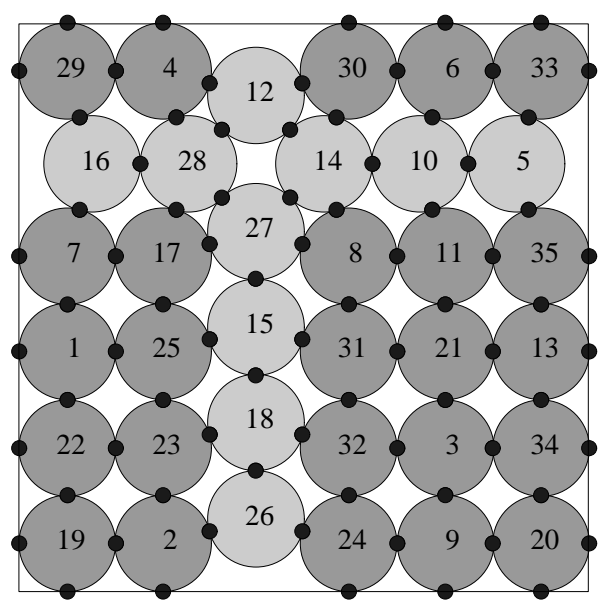

35 disks $(2,3)$

$\mathrm{m}=0.20276360086323 \quad 80$ bonds

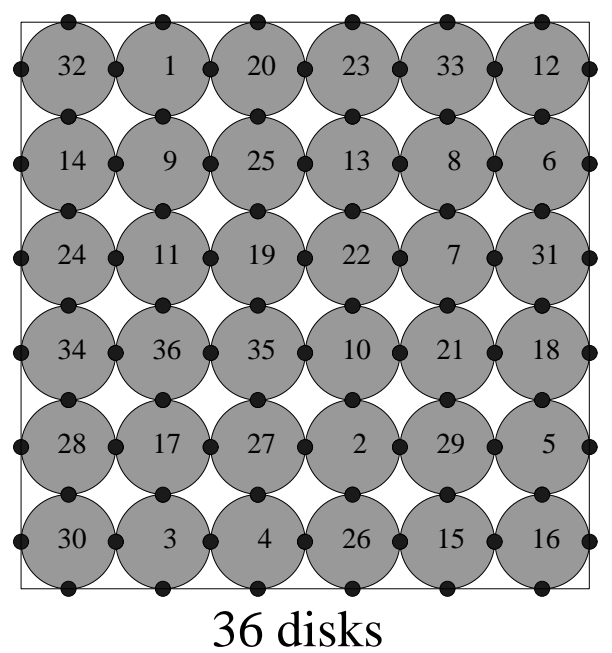

$\mathrm{m}=0.20000000000000 \quad 84$ bonds

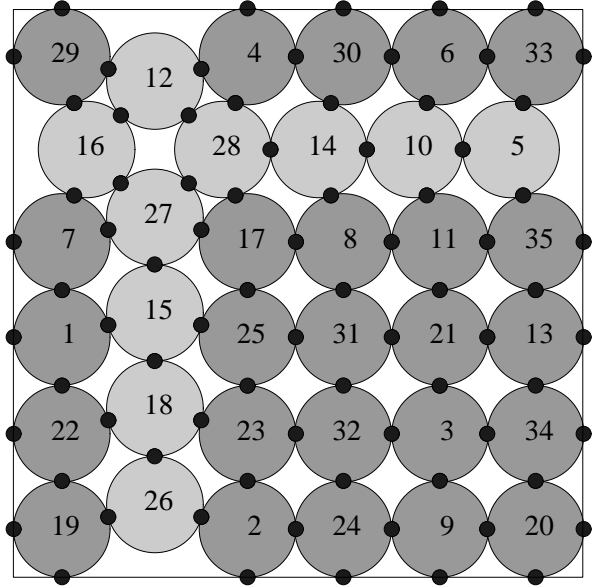

35 disks $(2,2)$

$\mathrm{m}=0.2027636008632380$ bonds

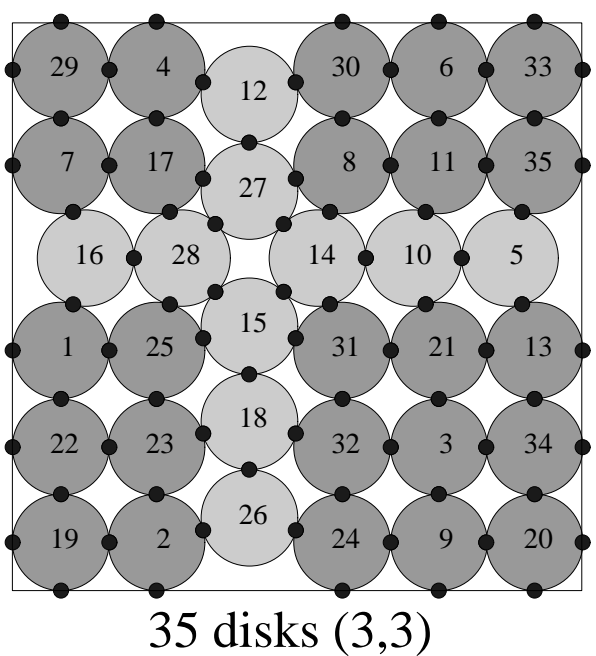

$\mathrm{m}=0.2027636008632380$ bonds

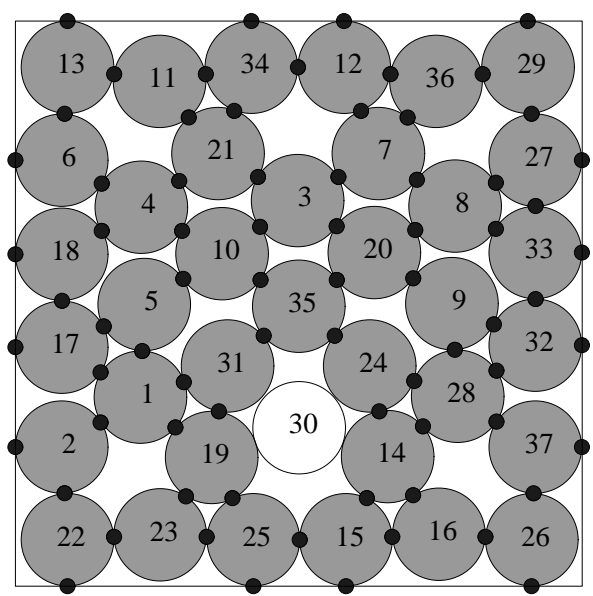

37 disks

$\mathrm{m}=0.19623810145141 \quad 73$ bonds

Figure 2.4: The densest packings found of 34 to 37 disks 


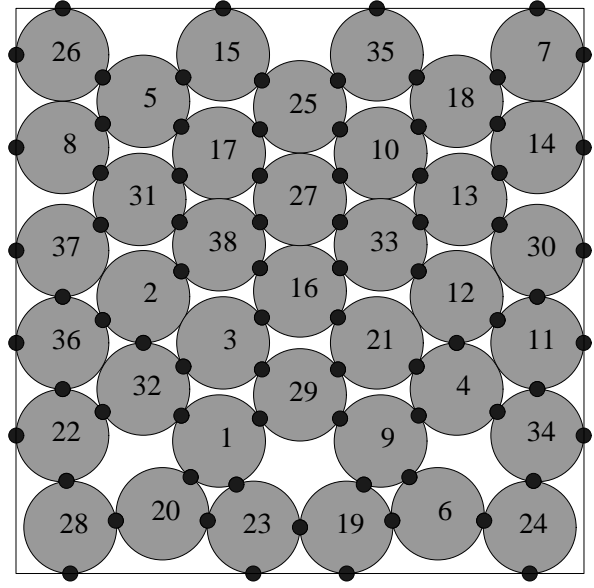

38 disks

$\mathrm{m}=0.19534230412691 \quad 77$ bonds

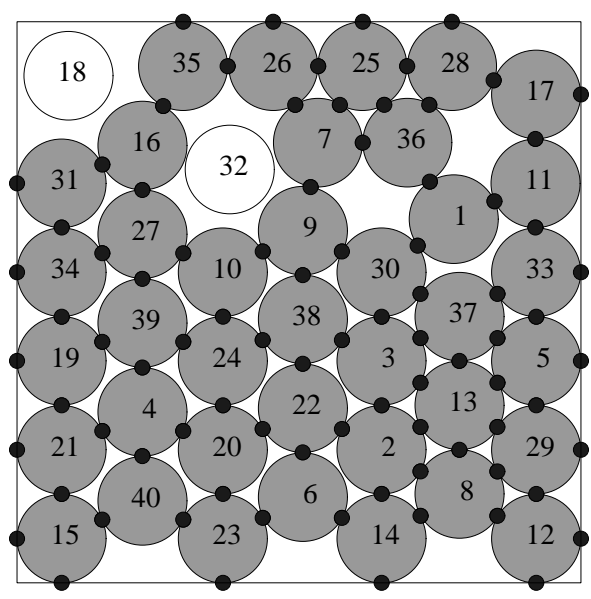

40 disks

$\mathrm{m}=0.1881755220183285$ bonds

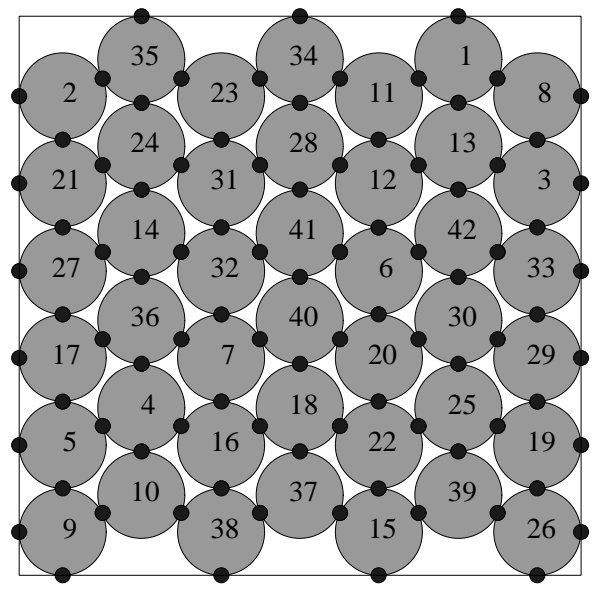

42 disks

$\mathrm{m}=0.18427707211710 \quad 90$ bonds

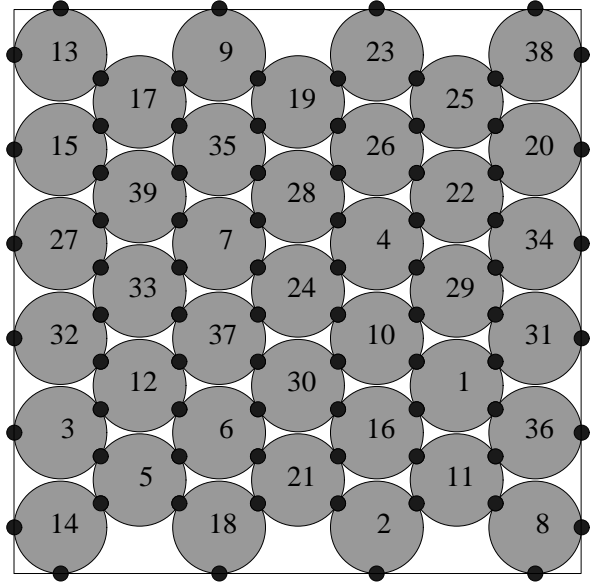

39 disks

$\mathrm{m}=0.1943650631615180$ bonds

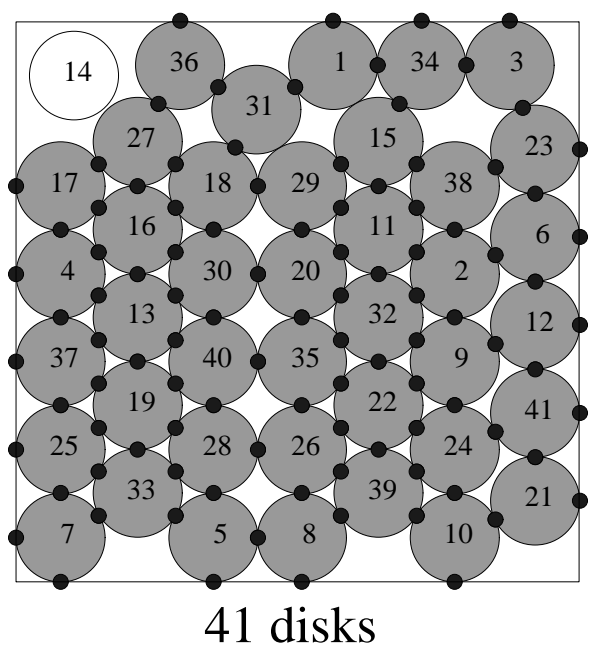

$\mathrm{m}=0.18609951184812100$ bonds

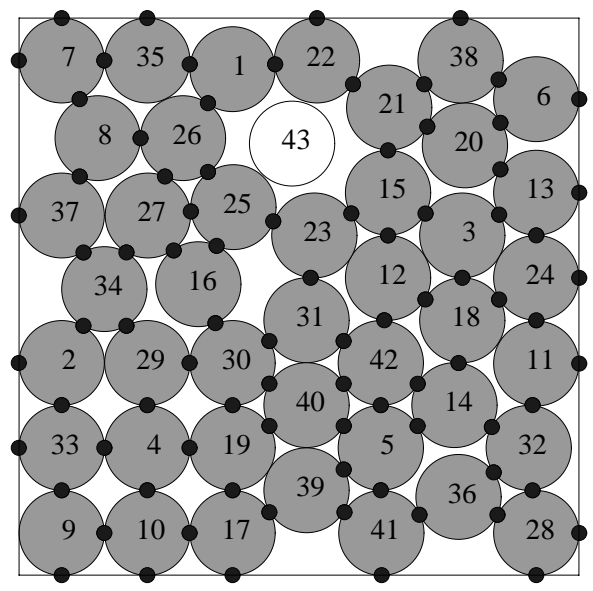

43 disks

$\mathrm{m}=0.18019113545743 \quad 85$ bonds

Figure 2.5: The densest packings found of 38 to 43 disks 


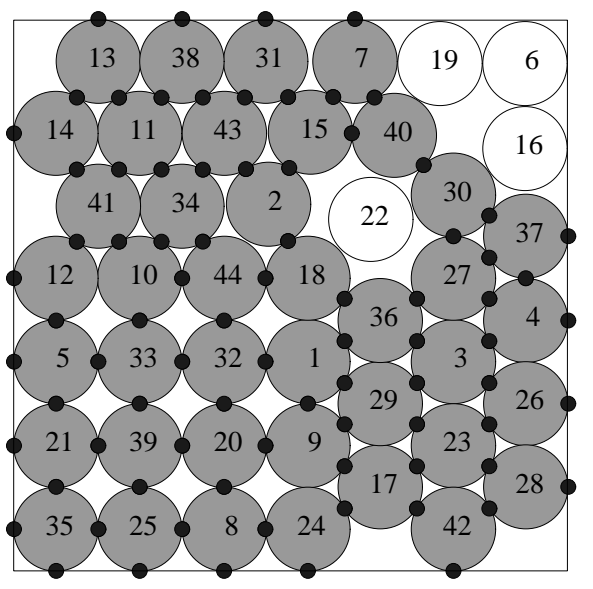

44 disks

$\mathrm{m}=0.17863924567120 \quad 82$ bonds

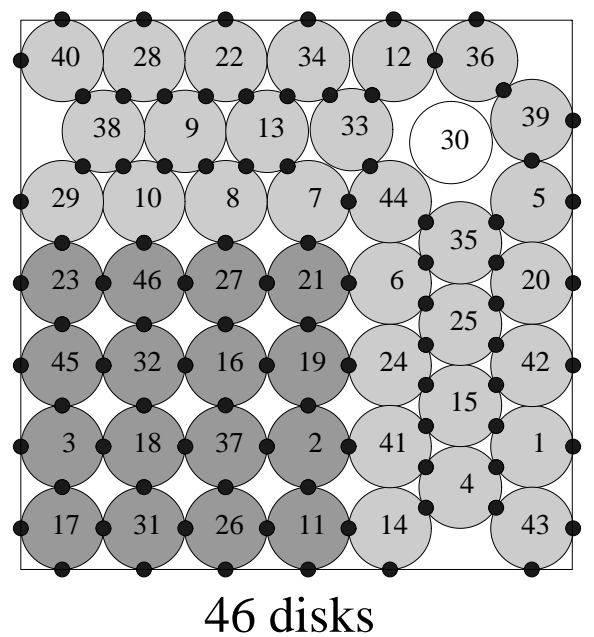

$\mathrm{m}=0.1744593608724191$ bonds

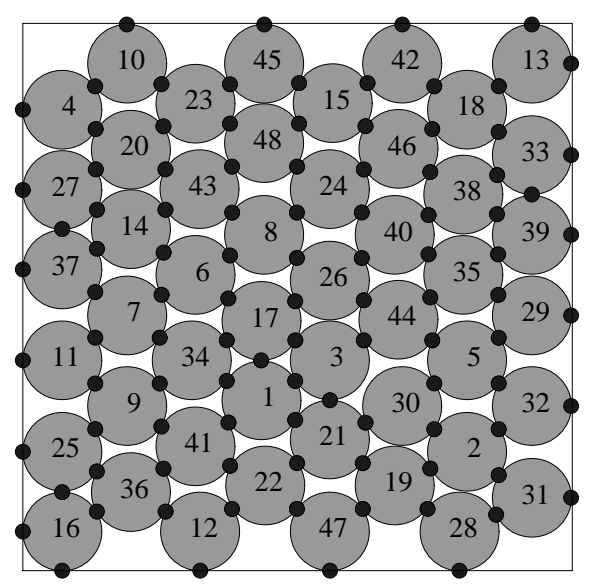

48 disks

$\mathrm{m}=0.16938210954876 \quad 101$ bonds

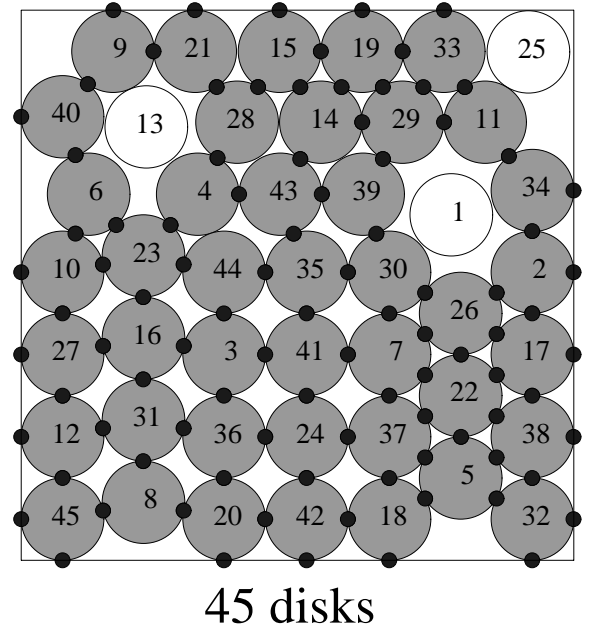

$\mathrm{m}=0.1757163141755994$ bonds

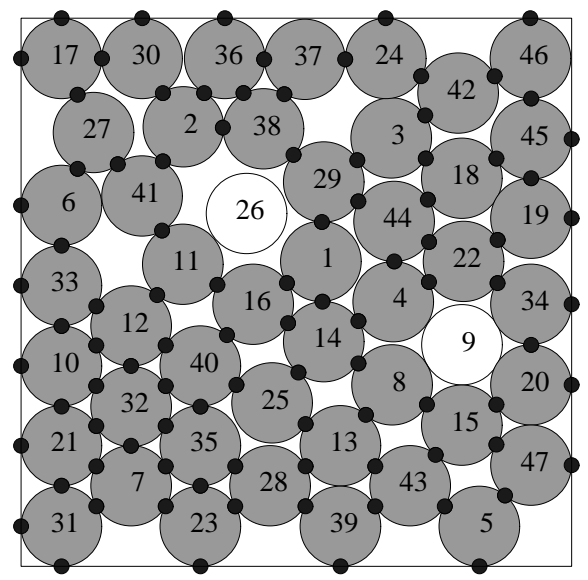

47 disks

$\mathrm{m}=0.1712683072114194$ bonds

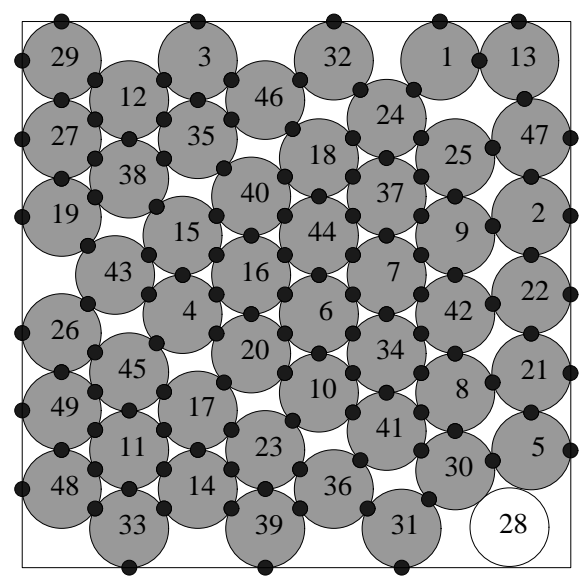

49 disks

$\mathrm{m}=0.16738607686833 \quad 120$ bonds

Figure 2.6: The densest packings found of 44 to 49 disks 

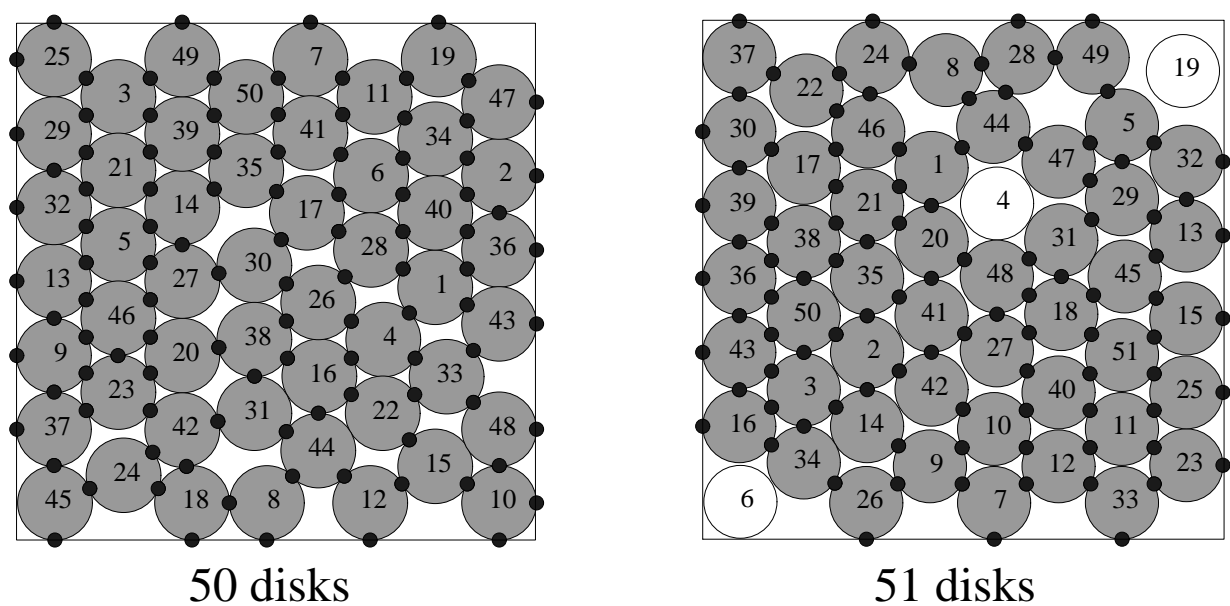

51 disks

$\mathrm{m}=0.16645462588286 \quad 104$ bonds

$\mathrm{m}=0.1656183743126099$ bonds

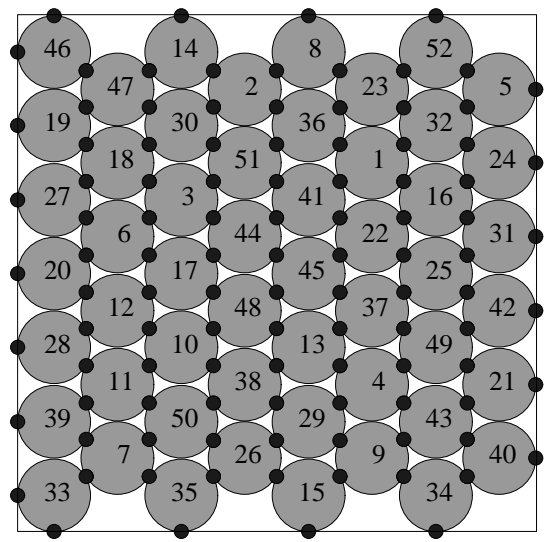

52 disks

$\mathrm{m}=0.16538623796964105$ bonds

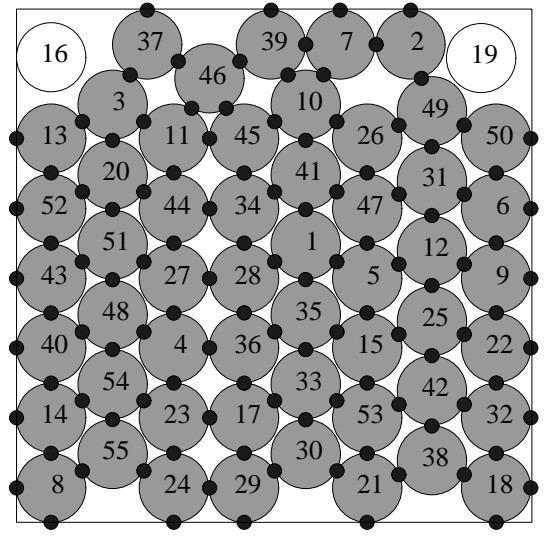

55 disks

$\mathrm{m}=0.15755574752972113$ bonds

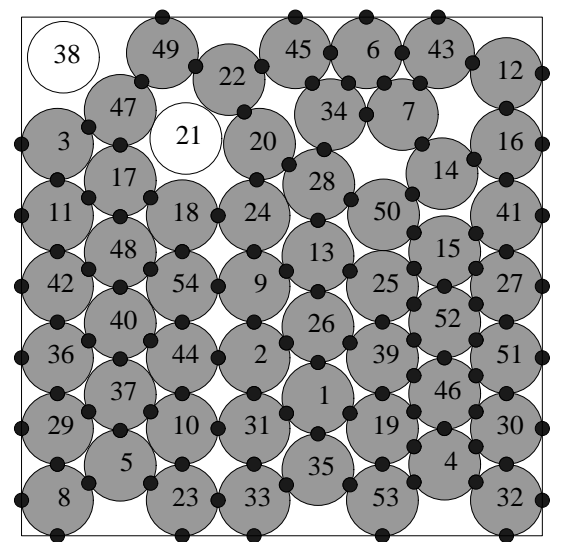

54 disks

$\mathrm{m}=0.15913951630719115$ bonds

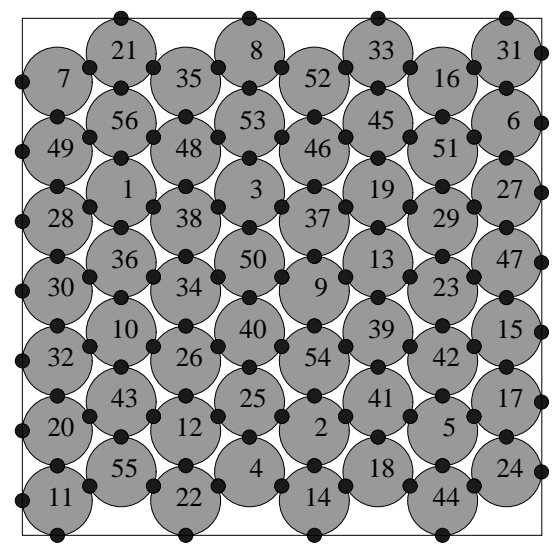

56 disks

$\mathrm{m}=0.15615650046215119$ bonds

Figure 2.7: The densest packings found of 50 to 52, and 54 to 56 disks 


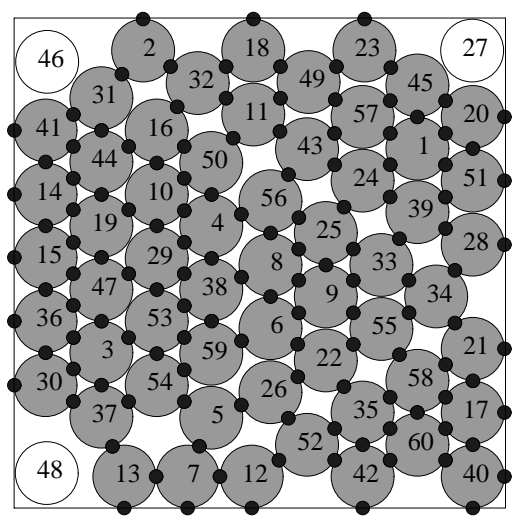

60 disks

$\mathrm{m}=0.14950565404867 \quad 129$ bonds

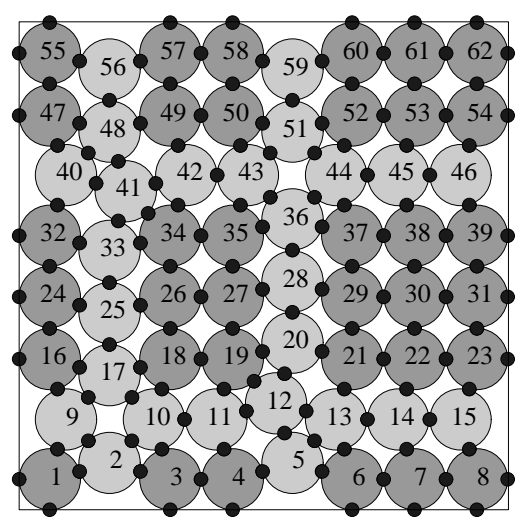

62 disks $(3,7 ; 2,5)$

$\mathrm{m}=0.14569394327531140$ bonds not the best packing

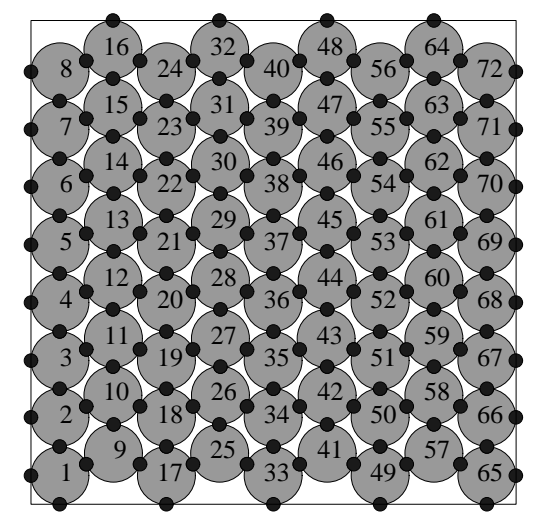

72 disks

$\mathrm{m}=0.13541666666667152$ bonds not the best packing

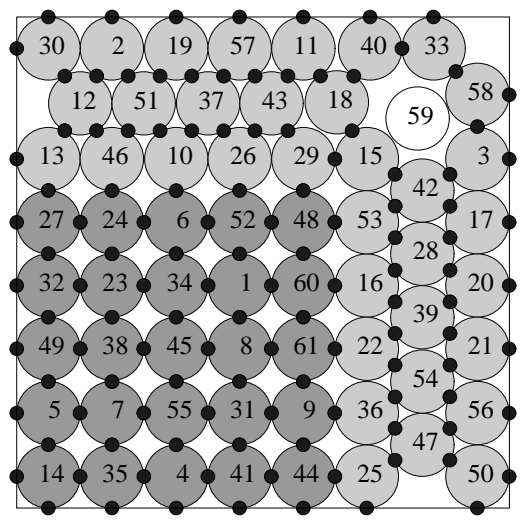

61 disks

$\mathrm{m}=0.14854412669518 \quad 121$ bonds

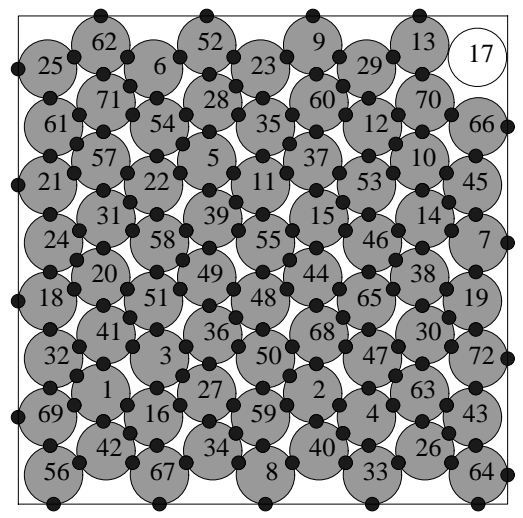

72 disks

$\mathrm{m}=0.13549029317569169$ bonds not the best packing

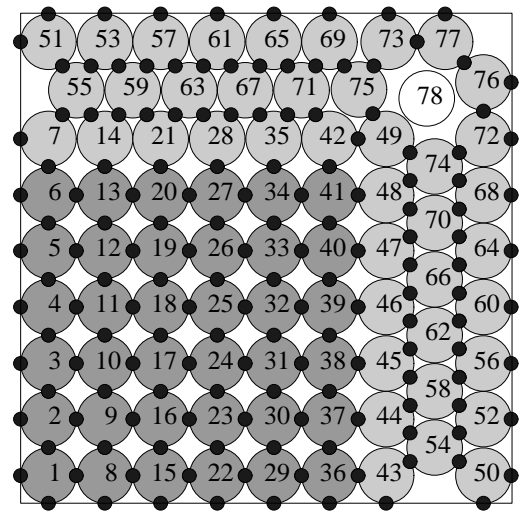

78 disks

$\mathrm{m}=0.12933240481510 \quad 155$ bonds not the best packing

Figure 2.8: The densest packings found of 60 and 61 disks and inferior packings of 62,72 , and 78 disks 

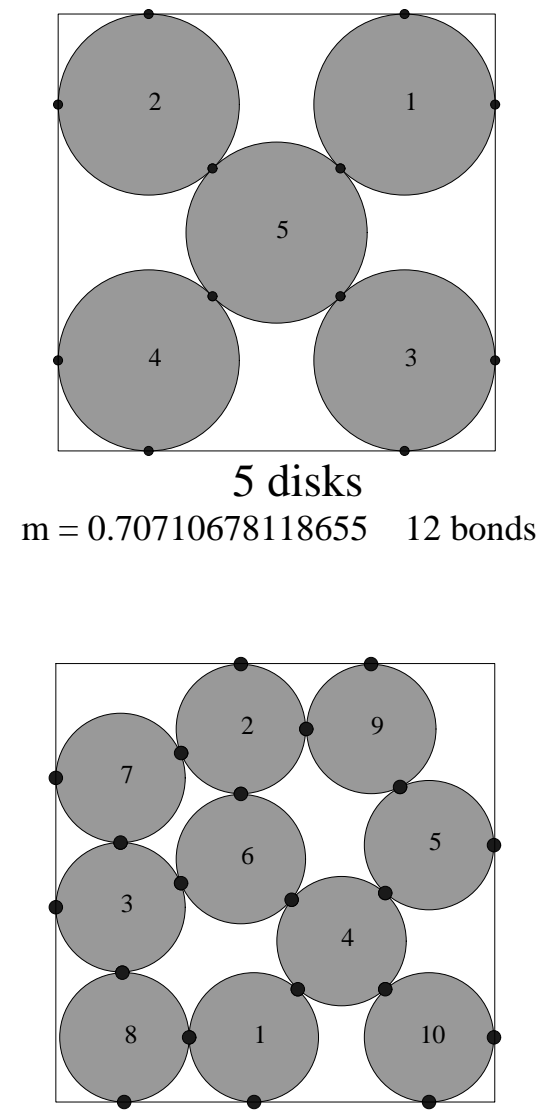

10 disks

$\mathrm{m}=0.4212795439839021$ bonds

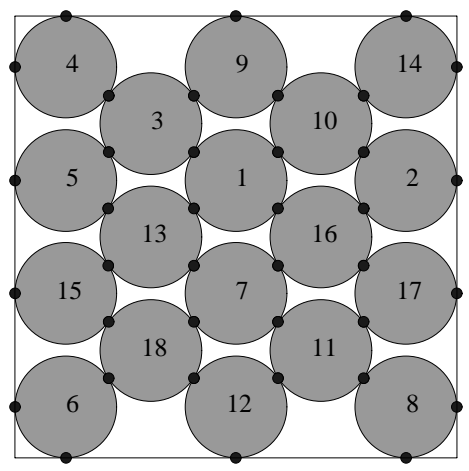

18 disks

$\mathrm{m}=0.30046260628867 \quad 38$ bonds

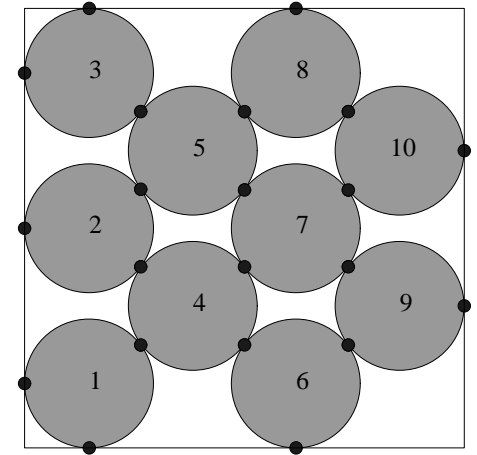

10 disks

$\mathrm{m}=0.41666666666667 \quad 21$ bonds not the best packing

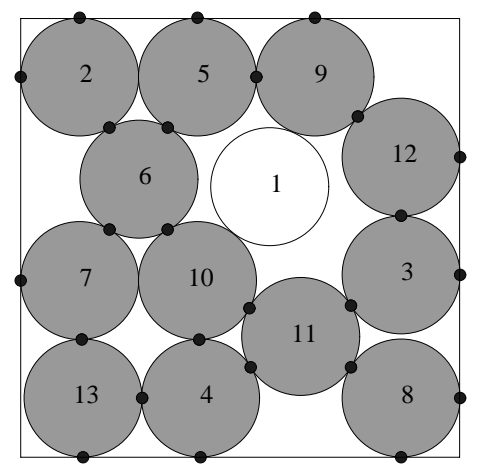

13 disks

$\mathrm{m}=0.3660960076964325$ bonds

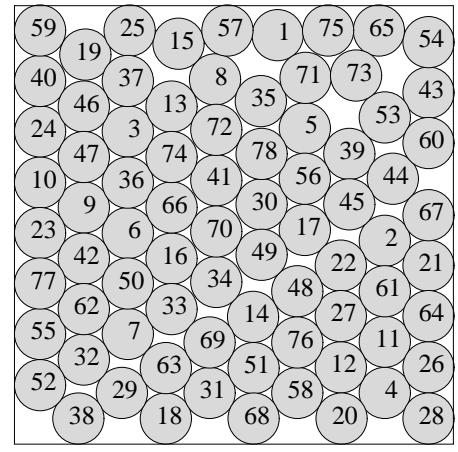

78 disks

$\mathrm{m}=0.13046077259640$ not a rigid packing

Figure 2.9: The densest packings of 5, 10, 13, and 18 disks, an inferior packing of 10 disks, and a challenger configuration of 78 disks 


\section{Series of packings with similar patterns}

$\mathbf{n}=\mathbf{k}^{\mathbf{2}}$. The packings of this series have an obvious square pattern with disk diameter $m=1 /(k-1)$. The pattern is optimal for $n=1,4,9$, and 16 , and probably 25 and 36 . For $k_{1}=7$ this yields value of $m=1 / 6$ which is smaller than the experimental diameter of the challenger packing of 49 disks in Fig. 2.6.

$\mathbf{n}=\mathbf{k}^{\mathbf{2}}-\mathbf{1}$. The pattern can be viewed as a square of $(k-1)^{2}$ disks arranged in straight rows and columns into which one row and one column of "shifted" disks are inserted. A "shifted" row or column each has $k-1$ disks. In the diagrams for $k=5(n=24$, Fig. 2.1) and $k=6(n=35$, Fig. 2.4), the "straight" disks are shaded more heavily than the "shifted" ones. Several equivalent packings are obtained by inserting the shifted row and column in different places among the straight ones. A pair of insertion position $(i, j), 2 \leq i, j \leq k-1$, identifies the packing, e.g., in packing of 24 disks (2,3) (Fig. 2.1), the shifted row is the second and the shifted column is the third (counting from the top left corner). Among the $(k-2)^{2}$ packings thus obtained, there are many congruent pairs. The pattern is fully developed at $n=8$ (one packing) and remains optimal for $n=15$ (one packing), $n=24$ (three equivalent packings), and $n=n_{0}=35$ (three equivalent packings). For $n_{1}=48$ the pattern looses its optimality, which can be shown as follows. The angle at the center of disk 13 in packing $(2,2)$ of 24 disks (see Fig. 2.1 ) in the triangle formed by centers of disks 13, 19 and 3 is $15^{\circ}$ with $\cos 15^{\circ}=.5 \sqrt{2+\sqrt{3}}$. Similar angles are the same in all the packings of the series which easily implies $m=1 /(k-3+\sqrt{2+\sqrt{3}})$. For $k_{1}=7$ this yields value of $m=0.168581424$ which is smaller than the experimental diameter of the challenger packing of 48 disks in Fig. 2.6.

$\mathbf{n}=\mathbf{k}^{\mathbf{2}}-\mathbf{2}$. This pattern is similar to the pattern of series $k^{2}-1$, only here there are two shifted rows and two shifted columns. The (non-optimal) packing of 62 disks depicted in Fig. 2.8 , shows this pattern for $k=8$. There are $(k-3)(k-4) / 2$ possible ways to insert a pair of shifted rows $\left(i_{1}, i_{2}\right)$ because of the restrictions $1<i_{1}<i_{2}<k$ and similarly for the columns $\left(j_{1}, j_{2}\right)$. Hence, there are $((k-3)(k-4) / 2)^{2}$ possible different index sets $\left(i_{1}, i_{2} ; j_{1}, j_{2}\right)$ for each of which we can construct an equivalent packing. Many of these are congruent. The pattern is fully developed at $k=5(n=23$, one packing $)$ and remains optimal for only one more value $k=k_{0}=6$ ( $n_{0}=34$, four equivalent packings.). Here $m=1 /(k-5+2 \sqrt{2+\sqrt{3}})$. For $k=k_{1}=7$ this yields value $m=0.1705406887$ which is smaller than that of the challenger packing of $n_{1}=47$ disks in Fig. 2.6.

$\mathbf{n}=\mathbf{k}^{\mathbf{2}} \mathbf{- 3}$. This pattern is represented by conjectured dense packings of 22, 33, 46, and 61 disks (Figs. 2.1, 2.3, 2.6, 2.8). A feature of the pattern is a (shaded more heavily on the pictures) densely packed "straight" square of $(k-3)^{3}$ disks in the bottom left corner. It follows that the pattern is not optimal for $k \geq 10(n \geq 97)$ because the "straight" square itself isn't. However, even for a smaller $n=n_{1}=78$ we were able to get a disk configuration 
that challenges the pattern as presented in Fig. 2.8, although this configuration is a not fully formed solid disk packing (see Fig. 2.9).

Looking at the (non-optimal) packing of 78 disks (see Fig. 2.8), the pattern can be further described as three alternating columns at the right and three alternating rows at the top with one rattler at the top right corner. In each of the three additional rows and the three additional columns, most disks are not touching each other. The exceptions are pairs 42-49 in the bottom additional row, 73-77 in the top additional row and 76-72 in the right additional column (Fig. 2.8). Also there is almost full contact for pairs of disks between adjacent additional rows and columns, except for the pair 42-75 which are not touching between the first and second additional rows. These features are identical in all the packings of the pattern.

Each such packing can be obtained by "tightening" a certain configuration $C$ described as follows: In $C$, disks 77 and 76 are not touching each other but all disks in additional rows and columns are touching their neighbors. ( $C$ is not a solid packing.) We take $C$ as an initial condition for the "billiards" packing algorithm [L], [LS]. To "tighten" the configuration, the "billiards" algorithm allows the disks to move chaotically in the square without overlaps while their diameter increases at a common rate until no further growth is possible. It is remarkable, that for each $n=k^{2}-3, k=5,6,7,8$ and 9 , this chaotic negotiation always converges (independently of the initial velocities) to the described pattern with parameter $m$ identical to double precision in all runs involving the same $n$. Moreover, when instead of the configuration $C$, we start with a random initial configuration and zero initial diameters of the disks, the same configuration with the same precision results in the runs that achieve the largest $m$, but only for $k=5,6,7$ and 8 , excluding value $k=9$. Also, for $k=4(n=13)$ which seems to be the smallest $n$ for which the pattern may exist, both methods, the one beginning with a zero-diameter random configuration and the one beginning with the configuration $C$, lead to the same packing which, however, deviates somewhat from the described pattern, see Fig. 2.9

$\mathbf{n}=\mathbf{k}(\mathbf{k}+\mathbf{1}) \quad$. The pattern consists of $k+1$ alternating columns with $k$ disks each, see, e.g., the packing of 30 disks in Fig. 2.2. Following the path of disks 2, 12, 6, 18, 3, 29 in this packing, we come to the expression $k \cos \alpha$ for the horizontal side of the square that contains the disk centers, where the angle $\alpha=\alpha(k)$ is that at the vertex 2 in the triangle $12,2,6$. The vertical side of the square is $k-1+\sin \alpha$, where the latter expression results from the path 12 , $2,5,30,24,7$. Equating these two expressions for the side we determine from the resulting equation: $\cos \alpha(k)=\left(k^{2}-k+\sqrt{2 k}\right) /\left(k^{2}+1\right)$ and $m=(k \cos \alpha(k))^{-1}$.

Looking again at the packing of 30 disks in Fig. 2.2, circles 1 and 2 are separated by a non-negative distance because $\alpha(k) \leq 30^{\circ}$ or $\cos \alpha(k) \geq \sqrt{3} / 2$ for $k=5$. The latter inequality holds and the pattern exists as a solid packing of non overlapping disks only for $k \geq 4$, i.e., for $n=20,30,42,56,72, \ldots$.

We show that the packing of the pattern is not optimal for $n \geq n_{1}=72$ as follows. We present another regular pattern of packings of $k(k+1)$ disks; for $k=8$ this alternative pattern is depicted in the second row and second column in Fig. 2.8 (with one rattler). The alternative 
pattern exists for all values of $n$ for which the main pattern exists. The diameter $\bar{m}=\bar{m}(k)$ of a disk in the alternative pattern is given by $\bar{m}(k)=1 /((k+1 / 2) \cos \beta(k)+(\sqrt{3} / 2) \sin \beta(k))$ where (in the example in Fig. 2.8) $\beta(k)$ for $k=8$ is the angle at disk 56 in the triangle 67,56 , 42. As before, we equate the horizontal and vertical sides of the square: $k \cos \beta+\cos (\beta+\pi / 3)=$ $(k-1) \sin (\beta+\pi / 3)+\sin \beta$. From this equation we easily determine $\beta=\beta(k)$ and then we find that $\bar{m}>m$ for all $n \geq n_{1}=72$ (but $\bar{m}<m$ for $n=20,30,42$, and 56). Note that the alternative packing, although it is better than the packing of the pattern for $n=72$ disks, is not optimal. For example, for 72 disks we found experimentally a disk configuration with an irregular structure (not shown) that is better than both the main or the alternative pattern.

$\mathbf{n}=\mathbf{k}^{\mathbf{2}}+\lfloor\mathbf{k} / \mathbf{2}\rfloor$. The pattern, as exemplified by cases $k=5(n=27$, Fig. 2.2$)$ and $k=6$ ( $n=39$, Fig. 2.5), consists of $k+1$ alternating columns, odd columns having $k$ disks each and even columns having $k-1$ disks each. As before, with $\alpha$ denoting the angle, say, at disk 15 in the triangle 7, 15, 10 in the packing of 27 disks in Fig. 2.2, we compute the side-length of the square obtained in two different ways: $k \cos \alpha=2(k-1) \sin \alpha$. From this equation we easily determine $\cos \alpha(k)$ and then $m=1 /(k \cos \alpha(k))$. The pattern exists when, as in the example of 27 disks (Fig. 2.2), disks 12 and 13 are separated by a non-negative distance. This occurs when $\sin \alpha(k) \geq 1 / 2$. Thus, the pattern exists only for $k=2,3,4,5,6$, and $7(n=5,10,18$, 27, 39, and 52) and those packings (shown in Figs 2.9, 2.2, 2.5, and 2.8) are, indeed, optimal, except the case of $n=10$ : proved [GMPW] for $n=5,10,18$ and conjectured for $n=27,39$, and 52.

For $k \geq 8$ the "ideal" pattern yields overlaps in pairs of disks. The overlap increases with $k$. For $k=8(n=68)$ the overlap is less than $1 \%$ of the disk diameter so it is naturally to expect that the optimal packing will be a small deviation from the main pattern. Experiments, indeed show that best configurations are of this sort. Unfortunately, none of them is a solid packing, because it becomes very difficult to find exactly in which pairs the disks are touching, and not merely just very close to each other.

\section{References}

[CFG] H. T. Croft, K. J. Falconer and R. K. Guy, Unsolved Problems in Geometry, Springer Verlag, Berlin, 1991, 107-111.

[GMPW] C. de Groot, M. Monagan, R. Peikert, and D. Wurtz, Packing circles in a square: a review and new results, in System Modeling and Optimization (Proc. 15th IFIP Conf. Zurich 1991), 45-54.

[FG] J. H. Folkman and R. L. Graham, A packing inequality for compact convex subsets of the plane, Canad. Math. Bull. 12 (1969), 745-752. 
THE ELECTRONiC JOURNAL OF COMBINATORICs 3 (1996), \#R16

[GL1] R. L. Graham and B. D. Lubachevsky, Dense packings of equal disks in an equilateral triangle: from 22 to 34 and beyond, The Electronic Journ. of Combinatorics 2 (1995), \#A1.

[GLNO] R. L. Graham, B. D. Lubachevsky, K. J. Nurmela, and P. R. J. Östergård, Packing congruent circles in a circle by stochastic optimization methods, (In preparation.)

[G] M. Goldberg, The packing of equal circles in a square, Math. Mag. 43 (1970), 24-30.

[L] B. D. Lubachevsky, How to simulate billiards and similar systems, J. Computational Physics 94 (1991), 255-283.

[LG1] B. D. Lubachevsky and R. L. Graham, Dense packings of $3 \mathrm{k}(\mathrm{k}+1)+1$ equal disks in a circle for $\mathrm{k}=1,2,3,4$, and 5 (Submitted for publication.)

[LS] B. D. Lubachevsky and F. H. Stillinger, Geometric properties of random disk packings, J. Statistical Physics 60 (1990), 561-583.

[MFP $] \quad$ C. D. Maranas, C. A. Floudas, P. M. Pardalos, New results in the packing of equal circles in a square, Discrete Mathematics 142 (1995), 287-293.

[NO] K. J. Nurmela and P. R. J. Östergård, Packing up to 50 equal circles in a square, Discrete \& Computational Geometry, submitted.

[O] N. Oler, A finite packing problem, Canad. Math. Bull. 4 (1961), 153-155.

[Sch] J. Schaer, On the packing of ten equal circles in a square, Math. Mag. 44 (1971), 139-140.

[Schl] K. Schlüter, Kreispackung in Quadraten, Elem. Math. 34 (1979), 12-14.

[Val] G. Valette, A better packing of ten circles in a square, Discrete Math. 76 (1989), $57-59$. 